\title{
Unilateral Urrets-Zavalia syndrome after Implantable Collamer Lens implantation: a case report and review of the literature
}

\author{
Duangratn Niruthisard and Ngamjit Kasetsuwan ${ }^{*}$ (])
}

\begin{abstract}
Background: Fixed dilated pupil after ophthalmic surgery or Urrets-Zavalia syndrome occurs after anterior segment surgery and usually relates to postoperative elevation of intraocular pressure. Urrets-Zavalia syndrome results in complaints of glare, halo, and photophobia. Retention of the viscoelastic agent during Implantable Collamer Lens implantation can result in postoperative elevation of intraocular pressure and Urrets-Zavalia syndrome. However, reversibility of pupillary dilatation is possible in some cases.
\end{abstract}

Case presentation: A 20-year-old Thai man with myopic astigmatism in both eyes underwent Implantable Collamer Lens implantation in the right eye. The preoperative slit-lamp examination of both eyes was normal, and no ectatic changes were detected from corneal tomography. One hour after the uncomplicated surgery of the right eye, intraocular pressure increased to $48 \mathrm{mmHg}$ and was immediately controlled with antiglaucoma medications. Postoperative pupillary dilatation was detected, presumably due to effect of preoperative application of mydriatic drops. At postoperative day 1 , the right pupil remained dilated but still reactive to light and pilocarpine $2 \%$ eye drops. Two weeks later, the left eye underwent the Implantable Collamer Lens implantation and showed neither postoperative increase in intraocular pressure nor postoperative pupillary dilatation. Two months after surgery, the dilatation of the right pupil partially reversed.

Conclusions: The findings of the right eye suggested diagnosis of Urrets-Zavalia syndrome. Compared with former reports, we noted an association between immediate control of elevation of postoperative intraocular pressure, light reactivity of the dilated pupil, and reactivity to pilocarpine $2 \%$ eye drops as potential predictors for reversibility of Urrets-Zavalia syndrome.

Keywords: Urrets-Zavalia syndrome, Implantable Collamer Lens implantation, Phakic intraocular lens implantation

\section{Background}

Fixed dilated pupil after ophthalmic surgery or UrretsZavalia syndrome (UZS) was first described following penetrating keratoplasty in keratoconus [1], and an association with postoperative elevation of intraocular pressure (IOP) was reported [2]. The majority of UZS cases occurred after penetrating keratoplasty in keratoconus [3,

*Correspondence: Ngamjitk@gmail.com

Department of Ophthalmology, Faculty of Medicine, Chulalongkorn

University, 1873 Rama 4 road, Pathumwan, Bangkok 10330, Thailand
4]. However, UZS was also reported to follow other anterior segment ophthalmic surgeries such as deep anterior lamellar keratoplasty (DALK) [5], Descemet's membrane stripping automated endothelial keratoplasty (DSAEK) [6], glaucoma surgeries [7-9], cataract surgery [10], and phakic intraocular lens implantation [11-16].

We reported a case of UZS following Implantable Collamer Lens (ICL) implantation. By reviewing previous literature, we attempted to identify possible risk factors of this syndrome and factors indicating reversibility of the dilated pupil. 


\section{Case presentation}

A 20-year-old healthy Thai man with myopic astigmatism attended our clinic for refractive surgery. He denied any past ophthalmic procedures. Best-corrected visual acuity (BCVA) was 20/32 in the right eye (OD) with $-7.25-3.75 \times 15^{\circ}$ and $20 / 40-1$ in the left eye (OS) with $-10.75-6.0 \times 170^{\circ}$. Corneal tomography and corneal thickness were obtained with a rotating Scheimpflug camera system for anterior segment analysis (OCULUS Optikgeräte, Pentacam, GmbH Wetzlar, Germany). The result showed that the thickness of the thinnest point of the right and left cornea was 521 and $511 \mu \mathrm{m}$, respectively, and no ectatic changes were detected. Instead of undergoing laser vision correction, we recommended implantation of toric Implantable Collamer Lens (toric ICL; STAAR Surgical, Nidau, Switzerland) in both eyes owing to thin corneas. Two separate operations of ICL implantation were scheduled, starting with the right eye. Preoperatively, the slit-lamp examination was completely normal. His pupils were reactive to light, and the diameters of pupils were equal. His scotopic diameters of the right and left pupils measured with a corneal topography (Carl Zeiss, ATLAS ${ }^{\mathrm{TM}} 9000$ Corneal Topographer, Jena, Germany) using PathFinder II Corneal Analysis Software were 7.0 and $6.4 \mathrm{~mm}$, respectively. The anterior chamber depth (ACD) measured with scanning slit corneal topography (Bausch \& Lomb, Orbscan II, Rochester, NY, USA) was $3.32 \mathrm{~mm}$ in the right eye and $3.2 \mathrm{~mm}$ in the left eye. The white-to-white (WTW) distance measured with a caliper was $11.5 \mathrm{~mm}$ in both eyes. The preoperative intraocular pressures of the right and the left eyes were 17 and $16 \mathrm{mmHg}$, respectively. The patient did not require preoperative preventive iridotomies because of the presence of a hole at the center of the optic part of the fourth generation of ICL. The preoperative endothelial cell counts of the right and left eyes were 2728 and
2824 cells $/ \mathrm{mm}^{2}$, respectively. The $12.6-\mathrm{mm}$ ICLs were selected for both eyes. Before surgery, tropicamide $1 \%$ ophthalmic solution (Novartis, Mydriacyl 1\%, UK) was applied until full pupillary dilatation was achieved. A viscoelastic agent (Alcon Laboratories, Inc., Provisc, Fort Worth, Texas, USA) was injected into the anterior chamber through a paracentesis port. The ICL was implanted in the right eye through a $2.75 \mathrm{~mm}$ temporal clear corneal incision. The viscoelastic agent was removed by manual irrigation with balance salt solution.

One hour after uncomplicated surgery, the patient complained of mild ocular pain. The IOP of the right eye measured with an applanation tonometer elevated to $48 \mathrm{mmHg}$. The cornea was clear, and the vault of ICL was 1.5 times of central corneal thickness (CCT). A $250 \mathrm{mg}$ acetazolamide tablet was given orally, and the IOP decreased to $30 \mathrm{mmHg}$ within 1 hour. The patient was then prescribed $250 \mathrm{mg}$ acetazolamide tablets every 6 hours before he was discharged. On the first postoperative day, IOP decreased to $15 \mathrm{mmHg}$. Visual acuity was 20/30-2 and improved to 20/25-2 with pinhole correction. Refraction showed $+2.5-1.75 \times 60^{\circ}$. Although the IOP was normal and the cornea was clear, we saw retention of viscoelastic agent in the anterior chamber, and the right pupil was $6 \mathrm{~mm}$ in diameter and slightly reactive to light. Although the ICL was in place with a vault 1.75 times of CCT, we decided to remove the viscoelastic agent by irrigation with balance salt solution. On the next day, the visual acuity was 20/25-1 and improved to $20 / 20-1$ with pinhole correction. Refraction showed $+1.37-2.25 \times 31^{\circ}$, and the IOP was $13 \mathrm{mmHg}$. The right pupil still dilated $6 \mathrm{~mm}$ in diameter. Five days after ICL implantation, the patient complained of glare especially at night despite satisfactory visual acuity. Despite normalized IOP, the right pupil remained $6 \mathrm{~mm}$ under slitlamp examination (Fig. 1a) and was slightly reactive to
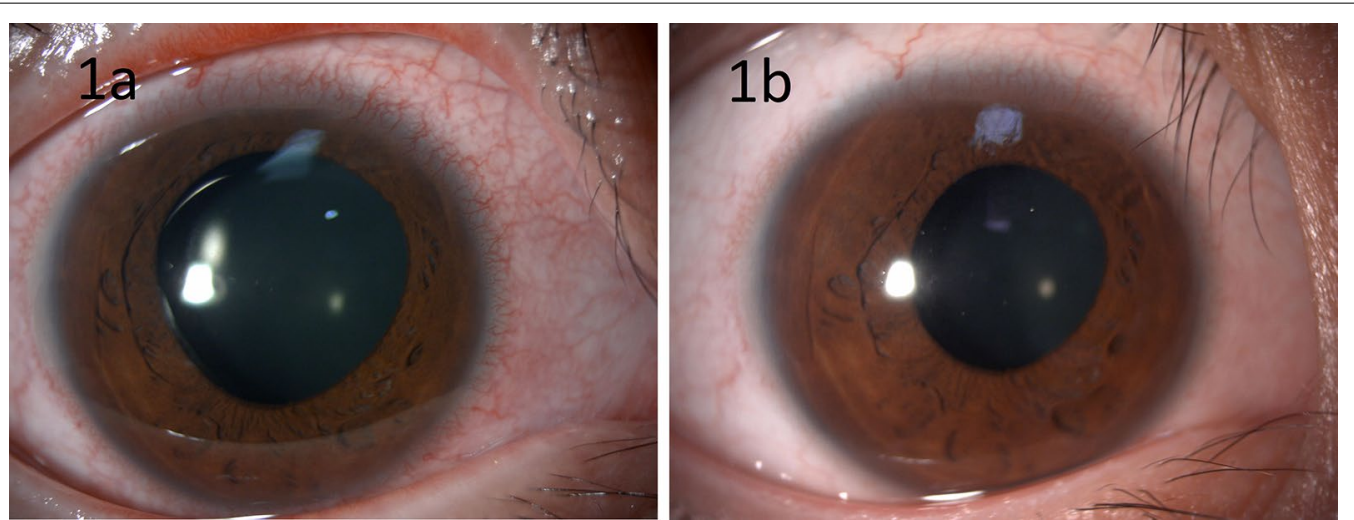

Fig. 1 This picture shows pupillary dilatation of the right eye at postoperative 5 days (a). Reactivity to pilocarpine $2 \%$ eye drops resulted in partial reversibility of pupillary dilatation $(\mathbf{b})$ 
light. Pilocarpine $2 \%$ eye drops (Alcon Laboratories, Inc., USA) were applied to the right eye every 15 minutes four times, and the pupil constricted to $3 \mathrm{~mm}$. The patient had applied pilocarpine $2 \%$ eye drops in the morning for 2 consecutive days and complained of less glare. Therefore, we advised him to continue pilocarpine $2 \%$ eye drops once a day in the morning. The visual acuity was $20 / 25+2$, the autorefraction was $-1.00-2.50 \times 45^{\circ}$, and IOP was $16 \mathrm{mmHg}$. The pupil was $4 \mathrm{~mm}$ in diameter and reactive to light (Fig. 1b). The ICL was in a good position with the 1.5-time CCT vault. We performed the ICL implantation in the left eye 2 weeks later. The surgery was successful without postoperative increased intraocular pressure, and his left pupil did not dilate after surgery.

Two months after surgery, the patient stopped pilocarpine $2 \%$ eye drops owing to conjunctival injection. He visited our clinic 3 months after surgery and claimed that the night glare improved, although more glare was detected in the right eye compared with the left eye. However, no glare was experienced during the day. In general, he was satisfied with the results of surgery. On examination, his right eye was $20 / 20-2$, while his left eye was $20 / 20$. The intraocular pressure was $13 \mathrm{mmHg}$ in both eyes. The right pupil was $3.5 \mathrm{~mm}$ in diameter under slit-lamp examination and was reactive to light. The left pupil was $2.5 \mathrm{~mm}$ in diameter (Fig. 2). The higher-order aberrations in root mean square (RMS) of his right and left eyes measured by corneal topography (Carl Zeiss, ATLAS $^{\text {TM }} 9000$ Corneal Topographer, Jena, Germany) using PathFinder II Corneal Analysis Software were 0.37 and $0.43 \mu \mathrm{m}$, respectively. No area of iris atrophy was detected in the right eye.

\section{Discussion}

Although the pathogenesis of UZS is controversial, there are two widely accepted mechanisms: iris ischemia and atrophy $[3,5,6,14,17,18]$ and injury to radial fibers of parasympathetic nerve [7]. Postoperative elevated IOP found in several anterior segment surgeries is a major cause of iris ischemia and atrophy. Tuft and colleagues reported UZS in three keratoconic patients who had elevation of IOP after penetrating keratoplasty and demonstrated delayed filling of iris vessels in isolated segments of the iris at 4-6 weeks postoperation by using anterior segment fluorescein angiography [2]. A summary of proposed causes of iris ischemia and atrophy can be found in Table 1.

Literature review revealed 6 cases of phakic intraocular lens implantation demonstrating UZS as summarized in Table 2. Postoperative increase in IOP was found in five cases of UZS after phakic intraocular lens implantation. In most of these cases ( 4 out of 5 cases), the elevation of
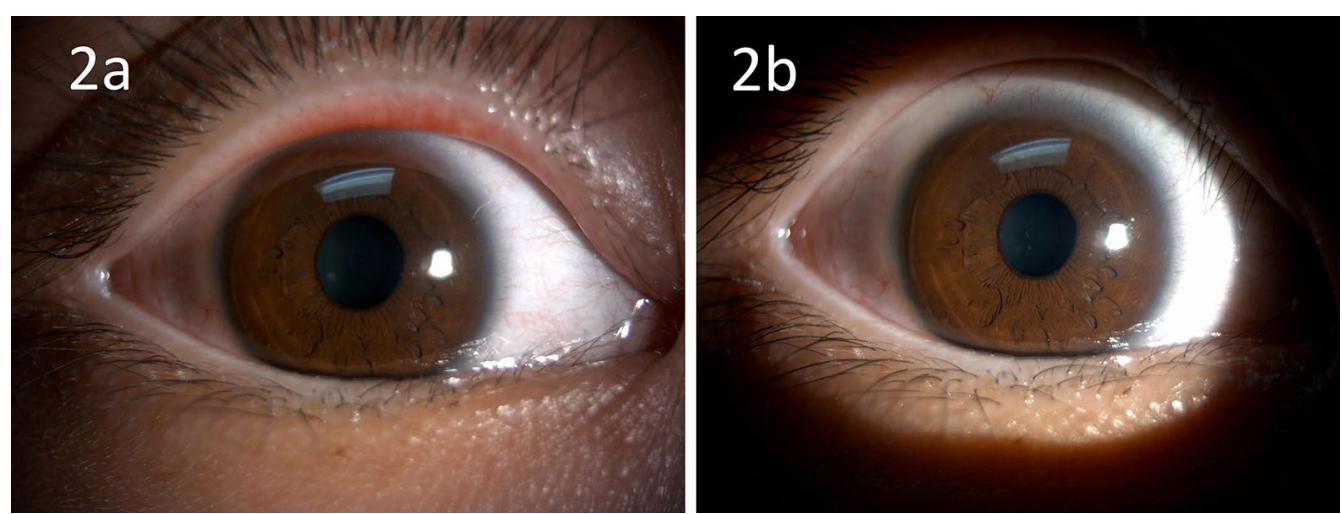

Fig. 2 The right pupil (a) at postoperative 2 months after cessation of pilocarpine 2\% eye drops for 1 month. The left pupil (b) after postoperative 2 months was also demonstrated for comparison

Table 1 Proposed causes of iris ischemia and atrophy resulting in Urrets-Zavalia Syndrome (UZS)

Increased intraocular pressure at postoperative period:

Retention of a viscoelastic agent after phakic IOL implantation [14]

Pupillary block due to air or gas bubble in the anterior chamber after anterior segment surgical procedures such as DALK, DSAEK, and intracameral

$\mathrm{C}_{3} \mathrm{~F}_{8}$ injection for treatment of acute corneal hydrops $[5,6,17]$

Direct trauma to iris during penetrating keratoplasty [18]

- Vascular strangulation caused by intraocular contents pushing lens-iris diaphragm against the edge of host cornea [3, 12]

Severe toxic anterior segment syndrome [19]

-Surgically induced hypromellose toxicity to vasculature or sphincter of iris [10] 


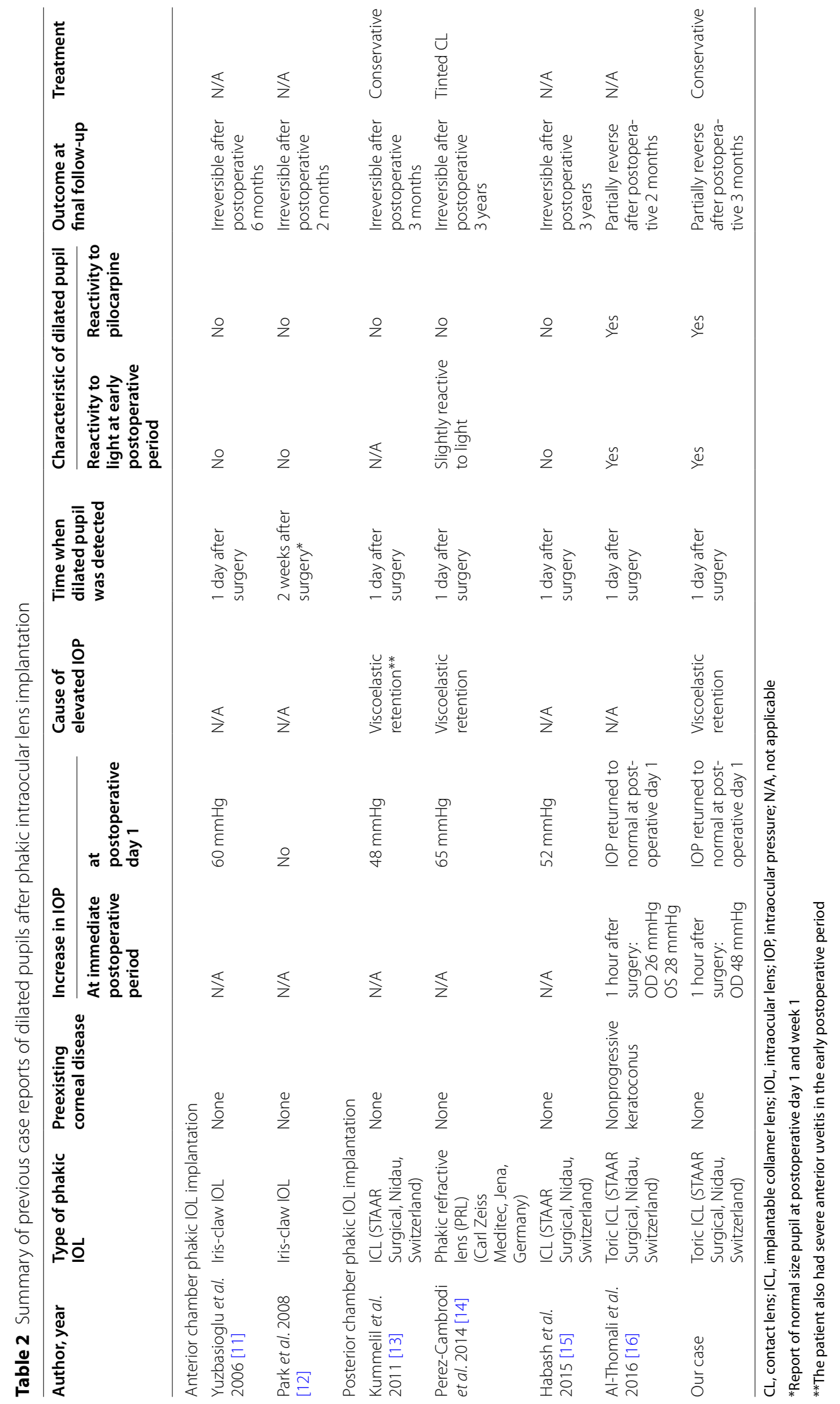


IOP was detected at postoperative day 1 , not at the immediate postoperative period, and all of the 4 cases experienced irreversible fixed dilated pupils [11, 13-15]. The intraocular pressure at postoperative day 1 ranged from 32 to $64 \mathrm{mmHg}$, and pupils were not reactive to pilocarpine $2 \%$ eye drops. In our case, the elevation of IOP was detected 1 hour after operation. The immediate vigorous treatment was initiated, and the IOP was normalized within 24 hours postoperation. The affected pupil was mid-dilated but still reactive to light and pilocarpine $2 \%$ eye drops. Three months after surgery, the pupillary dilatation partially reversed from $6 \mathrm{~mm}$ to $3.5 \mathrm{~mm}$. Our case demonstrated the reversibility of pupillary dilatation similar to the previous case reported by Al-Thomali and Alabhar [16] in which the elevated IOP was normalized in the immediate postoperative period. Reactivity of the dilated pupils to light and pilocarpine $2 \%$ eye drops was also noted in both our case and the case reported by AlThomali and Alabhar [16].

\section{Conclusions}

In summary, we report a case of UZS in phakic IOL implantation. Compared with previous reports, the data showed that aggressive IOP control in the immediate postoperative period tends to provide a chance for reversibility of dilated pupils. We also noted that postoperative reactivity of dilated pupils to light and pilocarpine $2 \%$ eye drops may be indicators for reversibility of pupillary dilatation.

\section{Acknowledgements}

Not applicable.

\section{Authors' contributions}

DN and NK took the patient history and performed the physical examination. DN performed collamer lens insertion in both eyes with the assistance of NK. DN wrote the initial draft of the manuscript. DN and NK reviewed and contributed to the final version of this case report. Both authors read and approved the final manuscript.

\section{Funding}

No funding was obtained to complete this case report.

\section{Availability of data and materials}

All data and material collected during this study are available from the corresponding author upon reasonable request.

\section{Declarations}

Ethics approval and consent to participate

Ethical approval was granted by the medical ethics committee of Faculty of Medicine, Chulalongkorn University, Thailand.

\section{Consent for publication}

Written informed consent was obtained from the patient for publication of this case report and any accompanying images. A copy of the written consent is available for review by the Editor-in-Chief of this journal.

\section{Competing interests}

The authors declare that they have no competing interests

Received: 3 January 2020 Accepted: 15 August 2021

Published online: 22 September 2021

\section{References}

1. Urrets Zavalia A Jr. Fixed, dilated pupil, iris atrophy and secondary glaucoma. Am J Ophthalmol. 1963;56:257-65.

2. Tuft SJ, Buckley RJ. Iris ischaemia following penetrating keratoplasty for keratoconus (Urrets-Zavalia syndrome). Cornea. 1995;14:618-22.

3. Davies PD, Ruben M. The paretic pupil: its incidence and aetiology after keratoplasty for keratoconus. Br J Ophthalmol. 1975;59(4):223-8.

4. Figueiredo GS, Kolli SS, Ahmad S, Gales K, Figueiredo FC. Urrets-Zavalia syndrome following penetrating keratoplasty for keratoconus. Graefes Arch Clin Exp Ophthalmol. 2013;251(3):809-15.

5. Bozkurt KT, Acar BT, Acar S. Fixed dilated pupilla as a common complication of deep anterior lamellar keratoplasty complicated with Descemet membrane perforation. Eur J Ophthalmol. 2013;23(2):164-70.

6. Fournie P, Ponchel C, Malecaze F, Arne JL. Fixed dilated pupil (UrretsZavalia syndrome) and anterior subcapsular cataract formation after Descemet stripping endothelial keratoplasty. Cornea. 2009;28(10):1184-6.

7. Espana EM, loannidis A, Tello C, Liebmann JM, Foster P, Ritch R. UrretsZavalia syndrome as a complication of argon laser peripheral iridoplasty. Br J Ophthalmol. 2007;91(4):427-9.

8. Walton DS. Urrets-Zavalia syndrome following goniotomy in a child. J AAPOS. 2013:17(1):114-5.

9. Klezlova A, Liebezeit S, Prokosch-Willing V, Gericke A, Pfeiffer N, Hoffmann EM. Urrets-Zavalia syndrome after combined trabeculotomy-trabeculectomy surgery. J Glaucoma. 2018;27(4):e80-3.

10. Tan AK, Humphry RC. The fixed dilated pupil after cataract surgeryis it related to intraocular use of hypromellose? $\mathrm{Br} J$ Ophthalmol. 1993;77(10):639-41.

11. Yuzbasioglu E, Helvacioglu F, Sencan S. Fixed, dilated pupil after phakic intraocular lens implantation. J Cataract Refract Surg. 2006;32(1):174-6.

12. Park SH, Kim SY, Kim HI, Yang SW. Urrets-Zavalia syndrome following iris-claw phakic intraocular lens implantation. J Refract Surg. 2008;24(9):959-61.

13. Kummelil MK, Nagappa S, Shetty A, “Urrets-Zavalia syndrome after implantation of implantable collamer lens" free paper presented at ASCRS Congress, San Diego, Califonia, March 2011.

14. Perez-Cambrodi RJ, Pinero-Llorens DP, Ruiz-Fortes JP, Blanes-Mompo FJ, Cervino-Exposito A. Fixed mydriatic pupil associated with an intraocular pressure rise as a complication of the implant of a Phakic Refractive Lens (PRL). Semin Ophthalmol. 2014;29(4):205-9.

15. Habash AA, Arfaj KA, Abdulsalam OA. Case report Urrets-Zavalia syndrome after implantable Collamer lens placement. Digit J Ophthalmol. 2015:21(3):1-11.

16. Al-Thomali TAAA. Unilateral mid-dilated reactive pupil after same session bilateral toric Implantable Collamer Lens implantation. Saudi J Health Sci. 2016;5(1):49-52.

17. Aralikatti AK, Tomlins PJ, Shah S. Urrets-Zavalia syndrome following intracameral C3F8 injection for acute corneal hydrops. Clin Exp Ophthalmol. 2008;36(2):198-9.

18. Spierer O, Lazar M. Urrets-Zavalia syndrome (fixed and dilated pupil following penetrating keratoplasty for keratoconus) and its variants. Surv Ophthalmol. 2014;59(3):304-10.

19. Huisingh C, McGwin G Jr. Cluster of Urrets-Zavalia syndrome: a sequel of toxic anterior segment syndrome. Br J Ophthalmol. 2013;97(12):1612.

\section{Publisher's Note}

Springer Nature remains neutral with regard to jurisdictional claims in published maps and institutional affiliations. 